Journal of Current and Advance Medical Research

January 2018, Vol. 5, No. 1, pp. 1-2

http://www.banglajol.info/index.php/JCAMR

ISSN (Print) 2313-447X

ISSN (Online) 2413-323X

DOI: http://dx.doi.org/10.3329/jcamr.v5i1.36536

EDITORIAL

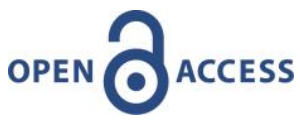

\title{
Prevention of Antimicrobial Resistance: Bangladesh Perspective
}

\author{
Md. Abdullah Yusuf
}

Assistant Professor, Department of microbiology, National Institute of Neurosciences \& Hospital, Dhaka, Bangladesh; Email: ayusuf75@yahoo.com; Cell no.: +8801817565830

Currently antimicrobial resistance (AMR) is a burning issue and it is one of the most significant threats to public health not only for Bangladesh but also for whole world. It becomes worst in the upcoming coming decades. As we know antimicrobial therapy is essential for treating and preventing bacterial infections, some of which can be life-threatening and is acquired as a result of critical medical interventions which includes surgery, chemotherapy and dialysis. However, the international rise in antimicrobial resistance has weakened our antibiotic armamentarium and multiresistant bacteria now cause over 150000 deaths annually in hospitals around the globe (WHO, 2013). Unfortunately, the evolution of drugresistant pathogens is unavoidable due to random genetic changes in the pathogens that can render antibiotics ineffective. Antibiotic therapy can succeed in killing susceptible pathogens and it also inadvertently selects for organisms that are resistant. Because each exposure to antibiotics contributes to this process, efforts to restrict antibiotic usage only slow the development of resistance. Ultimately, innovative antimicrobial drugs with diverse mechanisms of action will be needed to treat emerging resistant pathogens.

In England antibiotic consumption had been on the rise, with increased antibiotic prescribing fuelling increased bacterial resistance. Antimicrobial resistance (AMR) is therefore seen as one of the greatest health and public health challenges of our time. The consequences of AMR include increased treatment failure for common infections and decreased treatment options where antibiotics are vital. Antimicrobial stewardship is crucial to combating AMR and is an important element of antibiotic resistant strategy in Bangladesh, which has seven key areas for action, one of which is optimizing prescribing practice.
Inappropriate use of antibiotics contributes significantly to the acceleration of resistance. Furthermore, the overuse of antibiotics has been driven largely by their low cost and clinical effectiveness, which has led many patients to view them as cure-alls with few risks. Needlessly exposing patients to antibiotics for example, for viral or mild infections likely to resolve on their own, the use of overly broad-spectrum antibiotics and suboptimal doses of appropriate therapy hasten the evolution of resistant pathogens. While affordable, rapid and accurate point-of-care diagnostics are essential for determining appropriate therapy for many bacterial diseases, routine clinical use will be limited if the tests are too expensive or not accessible during routine clinical encounters. In the absence of a clear diagnostic result, many health care providers prescribe empiric broad spectrum therapy without knowing exactly what they are treating. Although inappropriate use is widespread in many parts of the world, where antibiotics are available without a prescription or oversight by a health care provider or stewardship team, overuse abounds even where antibiotic prescribing is more tightly regulated. Studies conducted in the USA indicate that around 258 million courses of antibiotics are dispensed annually for outpatient use (Hicks, 2013) and up to 75 per cent of ambulatory antibiotic prescriptions are for the treatment of common respiratory infections, which may or may not be bacterial in origin (McCaig, 1995).

This perception is reinforced by the fact that antibiotics are curative in nature and used for short durations. However, the clinical effectiveness of these drugs decreases over time, as resistance naturally increases, and this process is accelerated with inappropriate use. Moreover, there are numerous consequences associated with the use of 
antibiotics, including over 140,000 emergency department visits yearly in the USA for adverse incidents (mostly allergic reactions; CDC, 2013a). In addition, antibiotics can eliminate protective bacteria in the gut, leaving patients vulnerable to infection with Clostridium difficile, which causes diarrhoeal illness. It is estimated that antimicrobial resistance costs the US health care system over US\$20 billion annually in excess care and an additional $\$ 35$ billion in lost productivity (Roberts et al., 2009). The inappropriate use of antimicrobial drugs is particularly concerning because highly resistant pathogens can easily cross national borders and rapidly spread around the globe. In recent years, strains of highly drug-resistant tuberculosis, carbapenem resistant Enterobacteriaceae and other resistant pathogens have spread outside their countries of origin within several years of their detection. Because resistant bacteria are unlikely to stay isolated, stewardship efforts must be improved globally and international attention is needed to improve surveillance of emerging pathogens and resistance patterns. A major challenge for clinicians and regulators will be to find stewardship interventions that can be scaled-up and involve multiple stakeholders, including providers, drug manufacturers, health care purchasers (insurers), governments and patients themselves. Such interventions should include practical and cost effective educational programmes targeted towards providers and patients that shift expectations for antibiotic prescriptions to a mutual understanding of the benefits and risks of these drugs.

Educational programmes alone, however, will not be sufficient to lower prescribing rates to recommended levels. Pushing down the inappropriate use of antibiotics also warrants stronger mechanisms that leverage the critical relationships between the stakeholders. Antibiotic prescribing is one area where the forces of prescriber knowledge, patient expectations and the prescriber's perceptions of these expectations, and culture all collide together. Certainly, research has highlighted how difficult it is to capture the complex interactions that contribute to antibiotic prescribing for UTIs. The overall goal of antimicrobial stewardship is to optimize clinical outcomes whilst minimizing the unintended consequences of antimicrobial use. It is a multifaceted approach that includes policies, guidelines, education, monitoring and audit. It should not be seen as a separate issue to that of controlling healthcare associated infections, as both are required in healthcare institutions, therefore a whole-systems approach is required.

[Journal of Current and Advance Medical Research 2018;5(1):1-2]

Cite this article as: Yusuf MA. Prevention of Antimicrobial Resistance: Bangladesh Perspective. J Curr Adv Med Res 2018;5(1):1-2 